\title{
NO als Regulator neuronaler Motilität und Regeneration in einfachen Nervensystemen
}

\author{
Gerd Bicker und Michael Stern
}

\section{Zusammenfassung}

Stickstoffmonoxid (NO) ist als gasförmiger Botenstoff im Nervensystem bekannt. Es spielt eine Rolle bei synaptischer Plastizität, aber auch bei neuronalen Entwicklungs- und Regenerationsvorgängen. Wir untersuchen die Funktion von NO und seiner Signalkaskade über zyklisches GMP am Heuschreckenembryo. Dessen sich entwickelndes Nervensystem ist sehr gut für pharmakologische Manipulationen in Gewebekultur geeignet. Wir analysieren die zellulären Mechanismen der NO-Wirkung an drei Beispielen: 1. im peripheren Nervensystem beim Auswachsen von Pionierneuronen in der Antenne, 2. im zentralen Nervensystem bei der axonalen Regeneration serotonerger Interneurone nach Axotomie und 3. im enterischen Nervensystem bei der Migration von Neuronen, die den Darm-Nervenplexus bilden. In allen Fällen dient intern freigesetztes Stickstoffmonoxid bzw. die Synthese von zyklischem GMP als ein permissives Signal für den jeweiligen Entwicklungsvorgang. Kohlenstoffmonoxid (CO) moduliert als ein weiterer gasförmiger Botenstoff antagonistisch zu NO die Wanderung der Darmneurone. Experimente mit humanen Modellneuronen in Zellkultur weisen darauf hin, dass NO auch bei der Bildung des menschlichen Nervensystems Aspekte der Zellmigration reguliert.

\begin{abstract}
Nitric oxide (NO) is known as a gaseous messenger in the nervous system. It plays a role in synaptic plasticity, but also in neural development and regeneration. We study the function of NO and its signalling cascade via cyclic GMP in the grasshopper embryo. Its developing nervous system is well suited for pharmacological manipulations in tissue culture. We analyse cellular mechanisms of NO action in three examples: 1 . in the peripheral nervous system during antennal pioneer axon outgrowth, 2 . in the central nervous system during axonal regeneration of serotonergic neurons after axotomy, and 3. in the enteric nervous system during migration of neurons forming the midgut nerve plexus. In each case, internally realeased NO or NO-induced cGMP synthesis acts as a permissive signal for the developmental process. Carbon monoxide (CO), as a second gaseous messenger, modulates enteric neuron migration antagonistic to NO. Experiments on human model neurons in cell culture indicate that NO regulates cell migration also during human brain development.
\end{abstract}

Keywords: growth cone; insect embryo; cGMP; protein kinase G; carbon monoxide

\section{Ein gasförmiger Botenstoff und sein Rezeptor}

Stickstoffmonoxid (NO) ist ein reaktives Gas, das im Organismus als membranpermeabler Botenstoff von Zelle zu Zelle signalisieren kann. Es wurde zuerst als Endothelium-derived Relaxing Factor (EDRF) beschrieben, der nach einer Freisetzung aus Endothelzellen die Relaxation der glatten Gefäßmuskulatur bewirkt. Die Entdeckung dieses atypischen Transmitters auch im Gehirn vor etwa 20 Jahren stellte eine der größeren Überraschungen in den
Neurowissenschaften dar. Eine Informationsübertragung durch ein Gas, das auf Bedarf von einer Zelle produziert wird, durch die Membran diffundiert und die Funktion einer anderen Zelle reguliert, repräsentiert ein vollständig neuartiges Signalisierungsprinzip in dem durch seine strikten anatomischen Verbindungen charakterisierten Nervensystem. Die prominente Rolle von NO bei der Modulation synaptischer Plastizität und bei der Pathologie neuronaler Erkrankungen wurde bereits von Wolf (1997) in einem Neuroforumartikel dargestellt.
$\mathrm{NO}$ wird in Neuronen von einer $\mathrm{Ca}^{2+} / \mathrm{Cal}-$ modulin stimulierten NO-Synthase (NOS) gebildet (Abbildung 1). Einer der Signaltransduktionswege dieses reaktiven Gases ist seine Bindung an die Häm-Gruppe der löslichen Guanylylcyclase (Garthwaite 2008). Die resultierende Aktivierung löslicher Guanylylcyclasen (sGC) reguliert zelluläre Funktionen über die Synthese von cGMP (Abbildung 1). Da die Aktivierung der sGC durch NO im nanomolaren Bereich erfolgt, stellt die $\mathrm{NO} /$ cGMP-Signaltransduktion eine hochempfindliche Signalkaskade der zellulären Kommunikation dar (Garthwaite 2008).

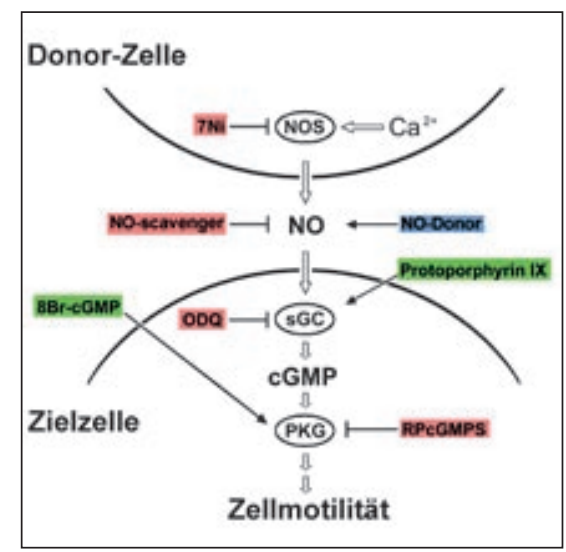

Abb. 1: Schema des transzellulären NO/ cGMP Signalweges.

Neuronale Aktivität der Donor-Zelle führt zum Einstrom von Kalzium, das zur Aktivierung der NO-Synthase (NOS) führt. NOS katalysiert die Bildung von Stickstoffmonoxid (NO), welches durch die Zellmembranen diffundiert und in der Zielzelle an die lösliche Guanylylcyclase (sGC) bindet. Die Stimulation von sGC durch NO führt zur Bildung des sekundären Botenstoffs zyklisches Guanosinmonophosphat (cGMP), welches wiederum die Proteinkinase G (PKG) aktiviert. Über weitere Zwischenschritte wird schließlich die Motilität von Zellen und Wachstumskegeln reguliert. In embryonaler Gewebekultur ist es möglich, an unterschiedlichen Stellen pharmakologisch in die Signalbahn einzugreifen, sei es durch Applikation von externem NO (blau), durch andere Aktivatoren der Signalbahn (grün) oder durch Inhibitoren (rot).

Zyklisches GMP aktiviert eine Vielzahl von Effektorproteinen einschließlich der Proteinkinase G (PKGI und PKGII), Phosphodiesterasen und durch zyklische Nucleotide regulierte Ionenkanäle (Lucas et al. 2000). Obwohl NO als ein unkonventioneller Transmitter durch die Zellmembran diffundieren kann, wird die Spezifität der zellulären Kommunikation über die aktivitätsabhän- 
gige Synthese und eine diskrete Verteilung des Rezeptorproteins (sGC) erhalten. Da die NO-Synthase NADPH als Kofaktor benötigt, lassen sich NO synthetisierende Zellen mit der NADPH-Diaphorase-Histochemie (Wolf 1997) auf mild fixierten Gewebeschnitten darstellen. Eine weitere Darstellungsmöglichkeit der NO-Donorzellen erfolgt mit Antikörpern gegen NO-Synthase. Die Zielzellen des NO-Signals können über eine NO-induzierte cGMP-Synthese im lebenden Gewebe mit einer immunzytochemischen Methodik identifiziert werden.

Auch Insekten nutzen das NO/cGMPSystem. Vor etwa 15 Jahren konnte die Freisetzung dieses Moleküls durch eine $\mathrm{Ca}^{2+} /$ Calmodulin stimulierte NOS in neuronalen Primärkulturen nachgewiesen und die cGMP synthetisierenden Zielzellen in einem Insektengehirn zytologisch dargestellt werden (Müller und Bicker 1994; Bicker et al. 1996). Inzwischen sind eine Reihe von zellulären Wirkungen der NO/cGMPSignaltransduktion bei der Modulation neuronaler Plastizität in den übersichtlichen Schaltkreisen von Insektennervensystemen

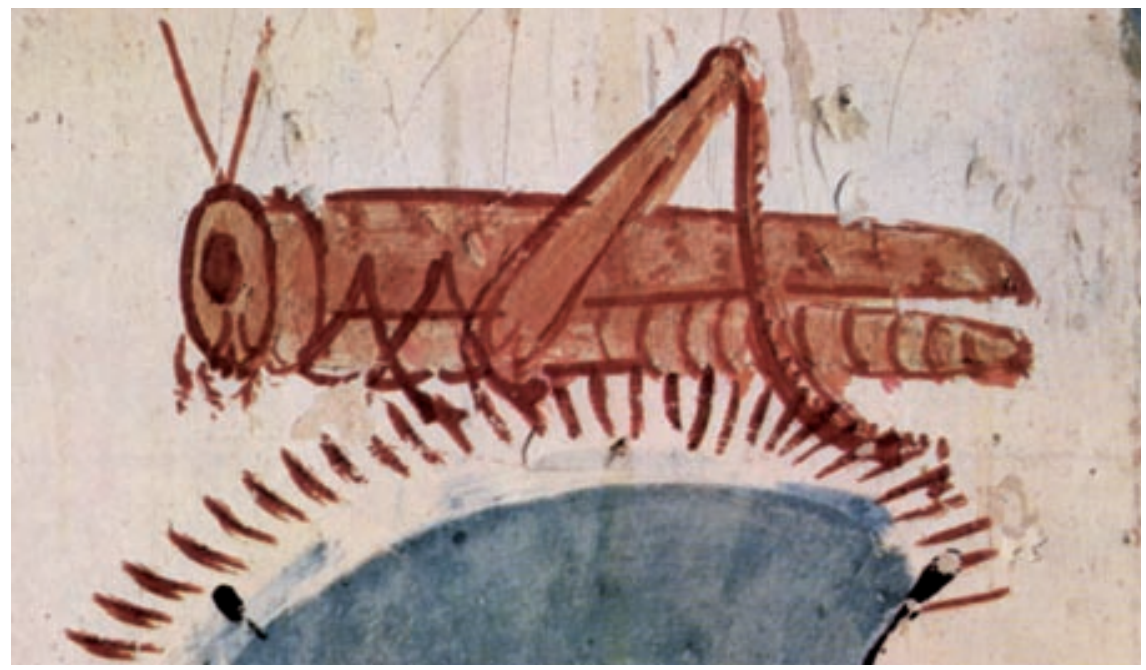

Abb. 2: Wanderheuschrecke auf Papyrusblüte. Ausschnitt aus einem Wandbild in der Grabkammer des Horemhab aus dem 15. Jh. v. Chr.

beschrieben worden. So wirkt NO zum Beispiel als retrograder Botenstoff bei der Lichtadaptation im visuellen System der Heuschrecke (Schmachtenberg und Bicker
1999) oder es stimuliert die Freisetzung von Neurotransmittervesikeln an der neuromuskulären Synapse von Drosophila-Larven (Wildemann und Bicker 1999).
HEKA Elektronik

Dr. Schulze GmbH

Wiesenstraße 71

D-67466 Lambrecht/Pfalz Germany

phone +49(0) $6325 / 9553-0$

fax $+49(0) 6325 / 9553-50$

eMailsales@heka.com

HEKA Electronics Inc. 47 Keddy Bridge Road R.R. \#2

Mahone Bay, NS BOJ 2EO

Canada

phone +19026240606

fax +19026240310

eMailnasales@heka.com

HEKA Instruments Inc. 2128 Bellmore Avenue Bellmore, New York 11710-5606 USA

phone +15168821155

fax +15164673125

eMailussales@heka.com

\section{Leadership}

International research and engineering

teams guarantee creativity and precision

for HEKA instruments and software.
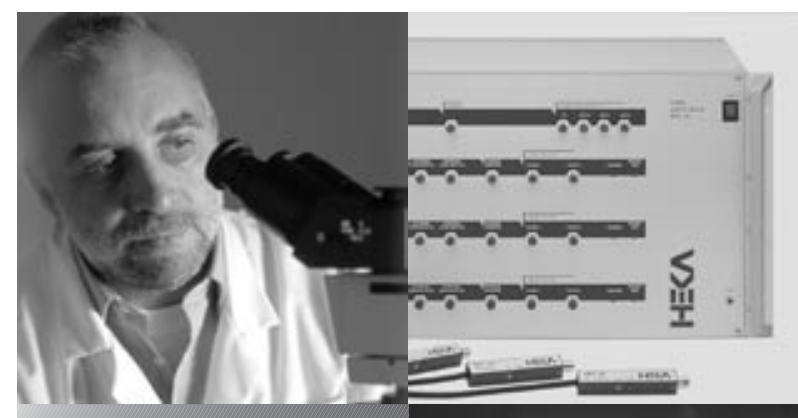

BLPC9 Amplifier $\quad$ ardx (SETUP) ON-CLL) (WHOLE-CLIL

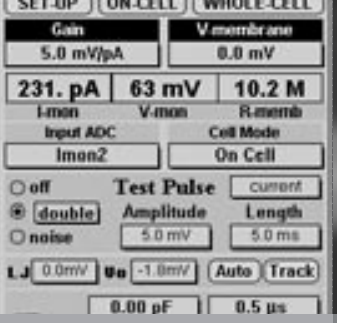

Electrophysiology

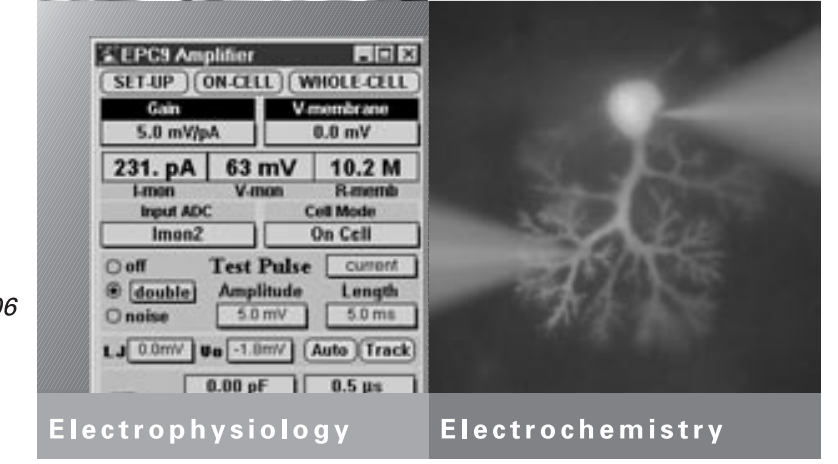

HEKA provides the finest instruments today to achieve the needed progress of tomorrow...

- patch clamp amplifiers

- multi-channel stimulation/ acquisition patch clamp systems

- potentiostats/galvanostats

- acquisition interfaces

- software for acquisition and analysis

- pipette pullers

- micromanipulators

- complete patch clamp set-ups

- scanning electrochemical microscopes

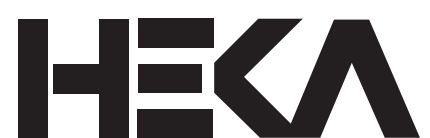




\section{NO als essenzieller Entwicklungsregulator}

Da die neuronale Expression von NOS im Wirbeltiernervensystem bei Entwicklungsund Regenerationsprozessen dynamisch reguliert wird, kann man NO nicht nur eine Rolle bei der Modulation synaptischer Plastizität, sondern vermutlich auch bei Entwicklungsmechanismen zuschreiben. Als Beispiel seien nur die Entwicklungsprozesse der Neurogenese, der neuronalen Migration und des Neuritenwachstums genannt, die durch experimentelle Änderungen in der NO-Konzentration moduliert werden können (Hess et al. 1993; Packer et al. 2003; Moreno-Lopez et al. 2004). Es stellt sich nun die Frage, ob NO ein essenzieller Faktor für die Entwicklung ist. Dazu wurde bei der genetisch zugänglichen Taufliege Drosophila, deren Genom nur ein einziges NOS-Gen (dNOS) enthält, mehrere Punktmutationen isoliert, die zu einem Ausfall der Enzymaktivität des mutierten NOS-Proteins führten (Regulski et al. 2004). Fliegen, die für eine der Punktmutationen homozygot sind, sterben während der embryonalen oder frühen larvalen Entwicklung. Natürlich lässt sich aus dieser Untersuchung noch kein Mechanismus ableiten, wie NO die Em- tenarten besitzen ein relativ übersichtliches zentrales Nervensystem, das aus individuell identifizierten Vorläuferzellen, Neuroblasten, entsteht, die sich im Stammzellmodus teilen. Seit einem Jahrhundert wird die Biologie der Heuschrecke intensiv erforscht, um Strategien zur Bekämpfung dieses schwarmbildenden Schadinsekts zu entwickeln, das bereits durch seine Bedrohung der Ernten im alten Ägypten (Abbildung 2) zum Symbol für eine biblische Plage wurde. Ironischerweise stellte sich bei der Forschung heraus, dass das Heuschreckennervensystem ein einfaches wie robustes neurophysiologisches Präparat für die Analyse komplexer sensomotorischer Integrationsprozesse ist. Die Bildung morphologisch eindeutig identifizierbarer Neurone aus den Neuroblasten ließen den Heuschreckenembryo außerdem zu einem Modellorganismus für das Studium zellulärer Mechanismen der axonalen Wegfindung werden. Im Lebenszyklus dieses hemimetabolen Schadinsekts legen die Weibchen die Eier als Gelege im Boden ab, die durch ein schaumartiges Sekret zusammengehalten werden. Der Embryo ernährt sich im Ei vom Dotter, schlüpft dann als wurmförmige Larve und entwickelt sich über fünf frei bewegliche Grashüpferstadien, die bereits eine große Ähnlichkeit zum erwachsenen Tier zeigen,
Wegfindung in Gewebekultur nachgewiesen (Kolodkin et al. 1992).

\section{NO/cGMP-Signaltransduktion reguliert das Auswachsen von Pionierneuronen}

Das Auswachsen der Nervenzellen während der Entwicklung wird von sogenannten Wachstumskegeln ermöglicht, die als bewegliche Zellstrukturen an der Spitze von neuronalen Fortsätzen sitzen. Pionierneurone legen die ersten axonalen Bahnen bei der Entwicklung des Nervensystems. Später auswachsende Axone können über Mechanismen der Zell-Zelladhäsion den bereits bestehenden Bahnen nachfolgen. Diese Strategie der axonalen Navigation wurde exemplarisch an Extremitätenanlagen des Heuschreckenembryos nachgewiesen, in denen identifizierbare Paare von Pionierneuronen die frühen axonalen Wege von Sinneszellen zum Zentralnervensystem festlegen, wenn die zu überbrückenden Distanzen noch kurz sind (Bate 1976; Bentley und O'Connor 1992). In der Extremitätenanlage befindet sich zwischen einem ektodermalen Epithel und lockerem mesodermalem Gewebe eine Basallamina, auf der die Axone von Sinneszellen und Motoneuronen auswachsen.

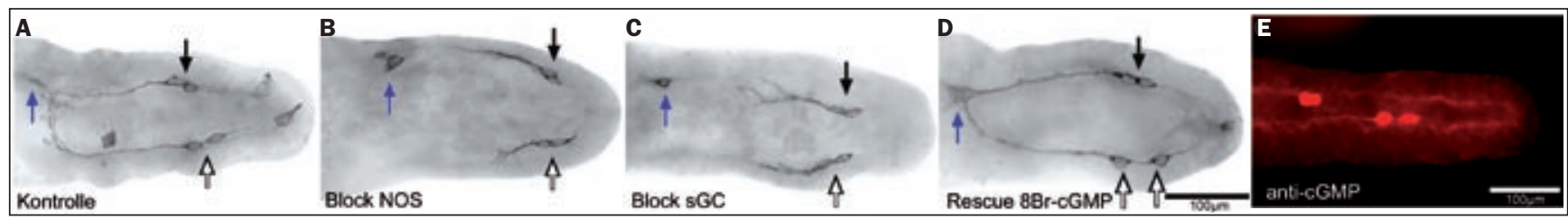

Abb. 3: Stickstoffmonoxid fördert das Auswachsen antennaler Pionierneurone im Heuschreckenembryo. (A-D) Antennenspitzen von 32\% Embryonen, nach 24h in Kultur, in denen ein neuronenspezifischer Marker immunzytochemisch dargestellt ist. Je zwei Paar Pionierneurone (schwarze und weiße Pfeile) sowie eine Wegweiserzelle an der Basis der Antenne (blaue Pfeile) sind angefärbt. In Kontrollversuchen (A) erreichen die Pionier-Axone während der Kulturdauer die Wegweiserzelle. Bei Blockierung der NOS durch Zugabe von 500 pM 7NI (B) oder Blockierung der SGC durch $200 \mu$ M ODQ (C) wird die Wegweiserzelle nicht erreicht. Im Rescue-Experiment wird diese hemmende Wirkung von $200 \mu \mathrm{M}$ ODQ durch gleichzeitige Zugabe von $500 \mu \mathrm{M}$ 8Br-cGMP vollständig wieder aufgehoben (D). Die cGMP-Produktion in den antennalen Pionierneuronen lässt sich nach Stimulation mit dem NO-Donor-Nitroprussid (SNP) in Gegenwart des Phosphodiesterase-Inhibitors IBMX mit einem Antiserum gegen cGMP nachweisen (E).

bryonalentwicklung reguliert. Die Letalität der NOS-Mutationen zeigt jedoch, dass bei Drosophila NO als ein essenzieller Entwicklungsregulator angesehen werden muss. Bei Säugetieren könnten die Auswirkungen von NOS-Defekten auf die Entwicklung durch die kompensatorische Wirkung mehrer NOS-Isoformen maskiert werden.

Um einen zellulären Zugang zur Rolle von NO bei der neuronalen Entwicklung $\mathrm{zu}$ bekommen, haben wir uns auf die Untersuchung der Wanderheuschrecke und der afrikanischen Wüstenheuschrecke (Locusta migratoria, Schistocerca gregaria) konzentriert. Diese sehr ähnlich gebauten Insek- zum adulten Insekt. Der Heuschreckenembryo lässt sich relativ leicht aus dem Ei herauspräparieren und intakt in Gewebekultur halten. Ein weiterer experimenteller Vorteil liegt darin, dass sich die ca. 50 Eier eines Geleges in unterschiedliche experimentelle und Kontrollgruppen gleichen Alters aufteilen lassen, die in Gewebekultur mit bioaktiven ,small molecule“ Verbindungen oder blockierenden Antikörpern behandelt werden können. So wurde zum Beispiel am Heuschreckenembryo das erste Mitglied der Semaphorine, einer prominenten Familie von Zellerkennungsmolekülen identifiziert und seine kausale Funktion bei der axonalen
Die Wachstumskegel der Pionierneurone navigieren dabei entlang von sezernierten Semophoringradienten. Weitere Leitsignale sind von epithelialen Zellen exprimierte transmembranständige Semaphorine und Wegweiserzellen, an denen sich die Filopodien entlang der Segmentgrenzen orientieren können. Abbildung 3 zeigt das Auswachsen zweier Paare von Pionierneuronen von einer distalen Region in der Antennenknospe in proximaler Richtung zum Cerebralganglion im Kopf. In späteren Entwicklungsstadien schließen sich die Axone von weiteren Sinneszellen an, die sich im ektodermalen Epithel differenzieren. 
Zum Auffinden von Embryonalstadien, in denen NO/cGMP gesteuerte Entwicklungsprozesse ablaufen, haben wir eine einfache zytochemische Technik eingesetzt, die von De Vente et al. (1987) erfunden wurde. Intakte Embryonen werden mit einem NO-Donor inkubiert, der die Bildung von cGMP in Zielzellen mit funktionell aktivierbaren sGC-Enzymen stimuliert. Der schnelle enzymatische Abbau des cGMP wird durch Gabe von PhosphodiesteraseInhibitoren verhindert. Nach Fixierung des Gewebes kann das gebildete cGMP mit einem Antikörper in diskreten Zellen nachgewiesen werden. Typischerweise tritt die cGMP-Immunreaktivität oftmals nur in bestimmten Entwicklungsstadien vorübergehend auf. Ein Beispiel für Zielzellen, die eine NO-induzierte cGMP-Immunreaktivität beim axonalen Auswachsen zeigen, sind die zwei Paare von Pionierneuronen an der Antennenspitze (Abbildung 3E). Da die epithelialen Zellen an der Basallamina den NOS-Marker NADPH-Diaphorase exprimieren, vermuten wir, dass NO als ein endogener Botenstoff freigesetzt wird, der als transzelluläres Signal auf die Pionierneurone einwirkt. Eine Inhibition der NO-Synthese durch einen NOS-Blocker (Abbildung 1) führt dosisabhängig zu einer Reduktion bis zur Verhinderung des Wachstums der Pionieraxone (Abbildung 3) (Seidel und Bicker 2000). Ein ähnlicher Effekt wird durch Hemmung der sGC erzielt. Um unspezifische Effekte der Enzyminhibitoren auf die Zellchemie des Embryos auszuschließen, konnten wir den Block der NOS durch 7NI und der sGC durch ODQ (Abbildung 1) durch eine gleichzeitige Applikation mit dem membranpermeablem cGMP-Analogon 8Br-cGMP vollständig aufheben (Abbildung 3D). Diese „Rescue-Experimente“ zeigen zudem, dass das cGMP-Signal nicht gerichtet auf den Wachtumskegel wirken muss, sondern eine homogene Badapplikation in der Gewebekultur ausreichend ist, um das vollständige Auswachsen der Pionieraxone zu gewährleisten (Seidel und Bicker 2000). Vermutlich wirkt zumindest in dem frühen Entwicklungsabschnitt an der Spitze der Antennenanlage die NO/cGMP-Kaskade als ein permissives und nicht als ein instruktives Signal für das axonale Wachstum. Weiterführende Untersuchungen zeigen, dass die Wachstumsregulation der Antennenpioniere kein Spezialfall ist, sondern auch bei anderen Pionierneuronen die Steuerung des axonalen Wachtums über NO/cGMP erfolgt (Pätschke und Bicker 2007).

\section{NO fördert Regeneration im Zentralnervensystem}

Wir fragten uns nun, ob die Wachstumsregulation durch NO auf das periphere Nervensystem beschränkt ist, oder ob sich ähnliche Vorgänge auch im Zentralnervensystem abspielen. Da die frühembryonale Entwicklung des Zentralnervensystems nur unter Schwierigkeiten in Gewebekultur nachvollzogen werden kann, haben wir diese Fragestellung mithilfe eines Regenerationsparadigmas untersucht (Stern und Bicker 2008). Die Regeneration des geschädigten Nervensystems steht seit jeher im Mittelpunkt medizinischen und neurobiologischen

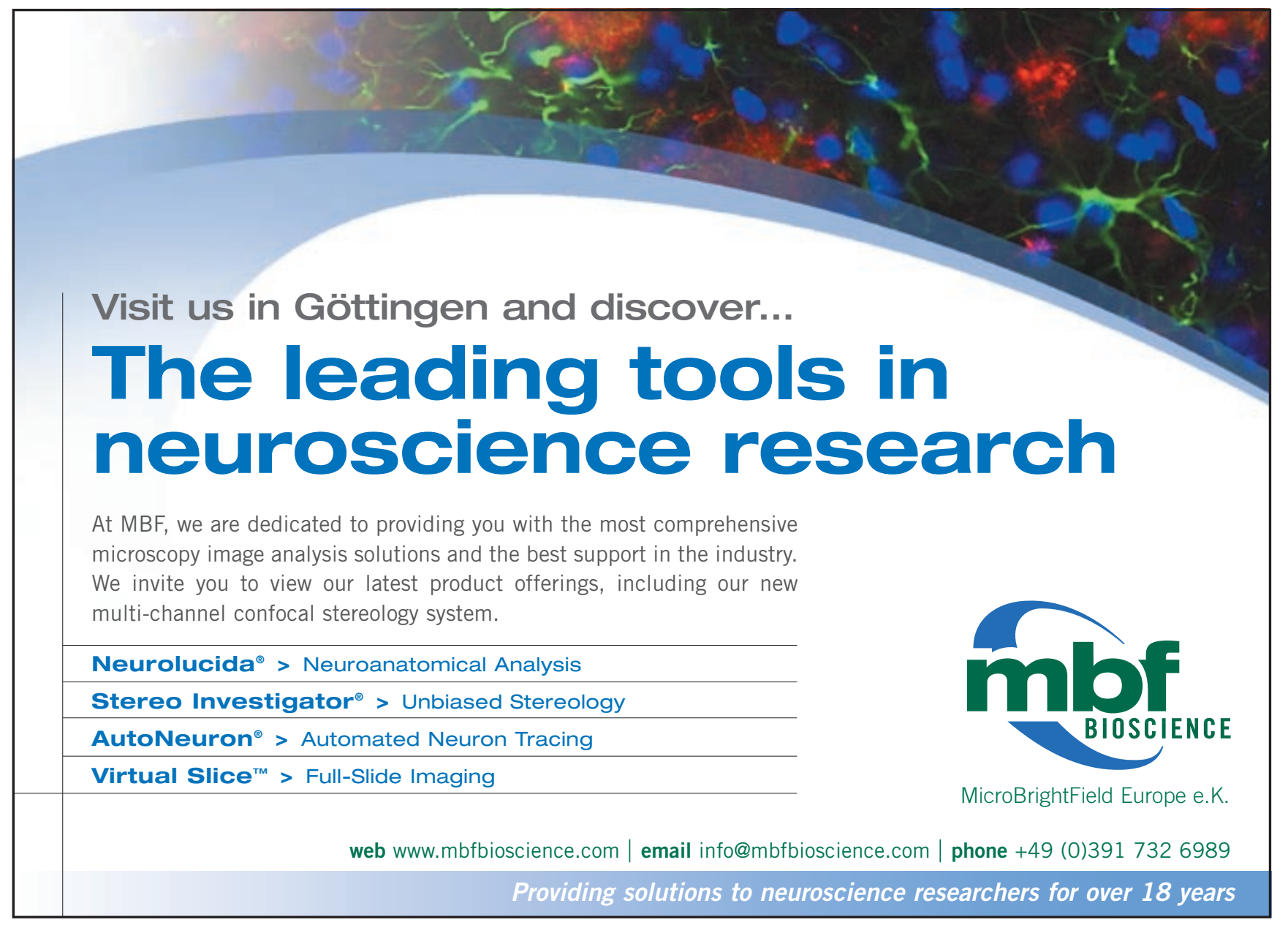




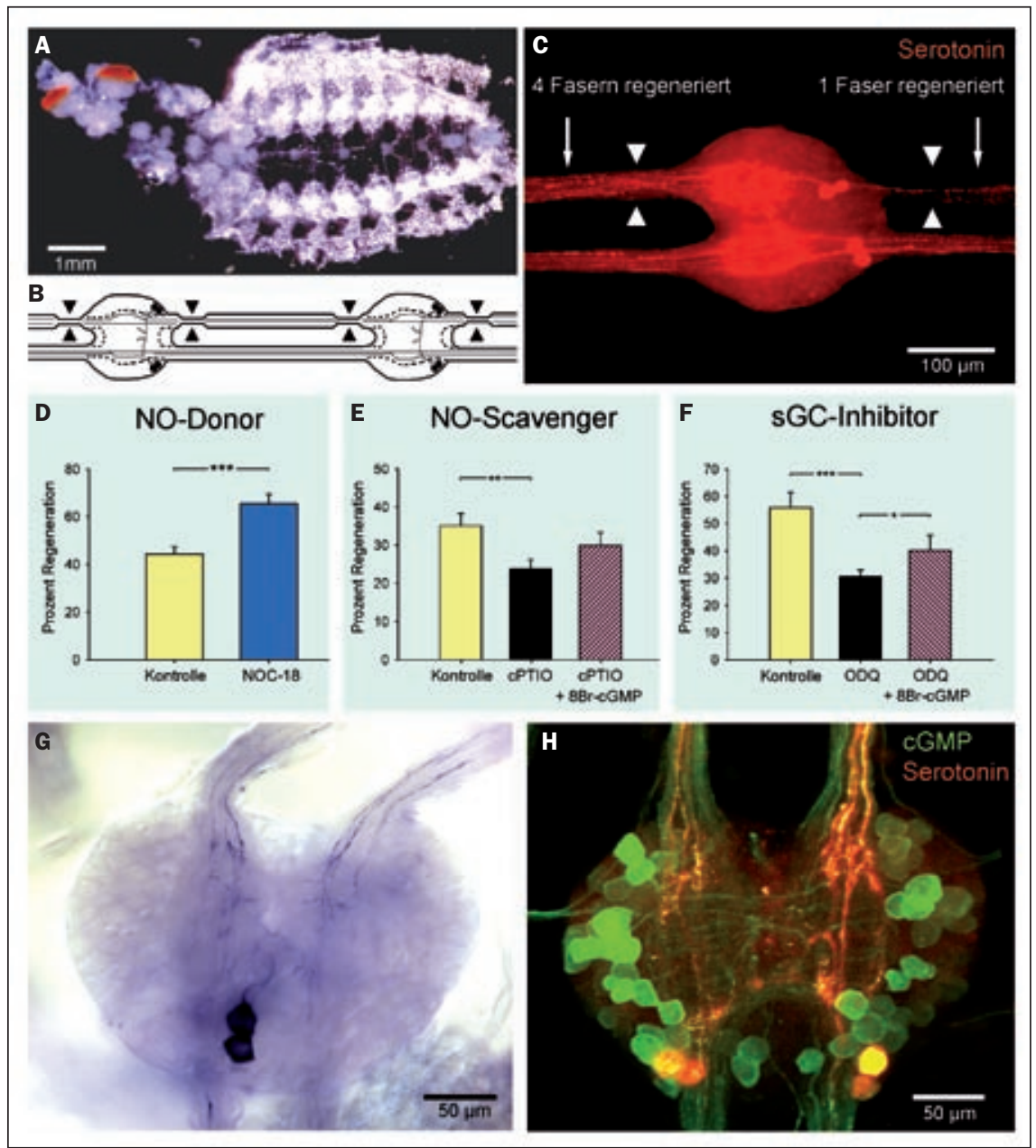

Abb. 4: Stickstoffmonoxid fördert die Regeneration serotonerger Axone im Heuschreckenembryo. (A) Filetpräparat eines zu 65\% entwickelten Heuschreckenembryos in Kultur. Das Zentralnervensystem ist freigelegt. (B) Eines der beiden Konnektive zwischen zwei Abdominalganglien wird an zwei Stellen gequetscht (Pfeilspitzen). Danach wird das Präparat für $48 \mathrm{~h}$ bei $30^{\circ} \mathrm{C}$ kultiviert. (C) Fluoreszenzfärbung der vier in jedem Ganglion vorhandenen intersegmentalen serotonergen Interneurone, $48 \mathrm{~h}$ nach dem Quetschen. (D) Unter Kontrollbedingungen regenerieren nach $48 \mathrm{~h}$ ca. $\mathbf{4 0 \%}$ der gequetschten Axone, in Gegenwart des NO-Donors NOC-18 (500 $\mu \mathrm{M})$ mehr als 60\%. (E) Abfangen von intern produziertem NO mit dem Scavenger cPTIO reduziert die Regeneration, dies lässt sich im Rescue-Versuch mit membranpermeablem cGMP (8Br-cGMP) teilweise aufheben. (F) Inhibition der NO-abhängigen SGC mit ODQ $(200 \mu \mathrm{M})$ reduziert Regeneration, dies wird im Rescue mit 8Br-cGMP wieder aufgehoben. Daten in (D-F) sind Mittelwerte aus mindestens zehn Embryonen. (G) NADPH-Diaphorase-Färbung zeigt, dass zu diesem Zeitpunkt NO produzierende Neurone vorhanden sind. (H) Konfokale Aufnahme einer Doppelfärbung gegen Serotonin und cGMP nach Stimulation mit einem NO-Donor. Viele Neurone antworten auf NO mit cGMP-Produktion, die serotonergen sind auch darunter.

Interesses. Während periphere Nerven nahezu problemlos regenerieren, ist zumindest bei höheren Wirbeltieren Regeneration im Zentralnervensystem (ZNS) nur sehr eingeschränkt möglich. Darum steht die Suche nach regenerationsfördernden Faktoren im Fokus aktueller Forschung. Dazu bietet es sich an, mit Wirbellosen zu arbeiten, bei denen Regeneration auch im ZNS möglich ist. Wir konnten bereits zeigen, dass Axone im adulten Heuschrecken-ZNS prinzipiell regenerieren (Pätschke at al. 2004). Noch besser lässt sich die Regeneration an einem Embryo-Kultursystem untersuchen. Dazu wird der späte Embryo geöffnet und mit freigelegtem ZNS in Kultur genommen (Abbildung 4A). Quetschen eines der paarigen Nerven, die die Ganglien des Bauchmarks verbinden, zerstört die darin verlaufenden Axone (Abbildung 4B). Mit immunzytochemischen Methoden kann man die Regeneration einzelner (z.B. serotonerger) Axone verfolgen (Abbildung 4C) und quantifizieren. Innerhalb von zwei Tagen regenerieren ca. $40 \%$ der Axone über die Quetschstelle hinaus.

Appliziert man Substanzen, die NO freisetzen, erhöht sich dieser Prozentsatz signifikant (Abbildung 4D). Nicht nur extern zugefügtes Stickstoffmonoxid, sondern auch intern produziertes kann Regeneration fördern: Fängt man intern produziertes NO mit einem NO-Scavenger $a b$, ist die Regeneration reduziert (Abbildung 4E). Als interne NO-Quelle kommen neben Hämozyten vor allem NOS exprimierende Neurone in Frage, die mit der NADPH-Diaphorase-Technik sichtbar gemacht werden können (Abbildung 4G). Deren Axone könnten durch die Quetschung zur NO-Produktion angeregt werden. Wie im peripheren Nervensystem erfolgt auch im ZNS die Wirkung von NO über cGMP. Wird die sGC inhibiert, ist die Regeneration reduziert, lässt sich aber im Rescue-Versuch durch Zugabe von $8 \mathrm{Br}-\mathrm{cG}$ MP teilweise wiederherstellen (Abbildung 4F). Die serotonergen Interneurone selbst, die hier als Beispiel betrachtet wurden, wie auch eine Vielzahl anderer Zellen vermag auf NO-Stimulation mit erhöhter cGMPProduktion zu antworten (Abbildung 4H), sodass man von einem allgemeinen Effekt ausgehen kann. Es ist möglich, und sogar wahrscheinlich, dass NO das Wachstum von ZNS-Axonen nicht nur bei der Regeneration, sondern auch bei der natürlichen Entwicklung beeinflusst.

Der Einfluss von NO auf Regeneration geschädigten Nervengewebes ist auch aus anderen Organismen bekannt, z.B. aus dem ZNS des Blutegels (Duan et al. 2005), aber dort wirkt NO indirekt über die Vermittlung von Mikroglia. Auch bei der Regeneration des peripheren Nervensystems von Wirbeltieren scheint es eine Rolle zu spielen (Keilhoff et al. 2002), hier allerdings durch Förderung der für Regeneration wichtigen vorhergehenden Wallerschen Degeneration.

\section{Zellmigration im enterischen Nervensystem}

Viele der molekularen Leitsignale, die axonales Auswachsen steuern, sind identisch mit den Leitsignalen, die für eine gerichtete Wanderung von Neuronen bei der Hirnentwicklung verantwortlich sind (Song und Poo 2001). Daher überlegten wir, ob das NO/ cGMP-System auch bei Insekten für die Regulation von Zellwanderung verantwortlich sein könnte. Wir untersuchten deshalb den 
als stomatogastrisches oder auch als enterisches bezeichneten Teil des peripheren Nervensystems, das den Darm innerviert. Die Entwicklung des enterischen Nervensystems von Insekten ähnelt der umfangreichen Zellwanderung aus den Neuralleisten der Wirbeltiere, bei denen sich Ganglien des autonomen Nervensystems bilden. Daher ist das enterische Nervensystem der Insekten ein gut etabliertes Modellsystem für zellbiologische Studien der neuronalen Wanderung (Hartenstein 1997). Der Darm der Heuschrecke besteht aus drei Abschnitten, dem Vorder-, Mittel- und Hinterdarm. Die Neurone des Mitteldarmplexus werden in einer neurogenen Zone des ektodermalen Vorderdarms geboren und bilden Zellpakete von postmitotischen, aber noch nicht vollständig differenzierten Neuronen an der Grenze zwischen Vorder- und Mitteldarm. Anschließend durchlaufen sie eine Phase schneller Zellwanderung, während der sie die Mitteldarmgrenze überqueren und sich als „Kettenmigration" auf vier geradlinig verlaufenden Zugstraßen über die Oberfläche des Mitteldarms bewegen (Abbildung 5A-C). Nach Beendigung dieser schnellen Wanderphase verlassen die Mitteldarmneurone die Zugstraßen, verteilen sich im Mitteldarmplexus und nehmen synaptische Kontakte mit der Darmmuskulatur auf (Abbildung 4D). Während der schnellen Wanderungsphase zeigen die Neuronen eine vorübergehende, NO-induzierte cGMP-Immunreaktivität, die mit dem Ausreifen des Plexus nachlässt (Haase und Bicker 2003). Die Bildung von cGMP wird daher entwicklungsabhängig reguliert und korreliert mit der Wanderungsphase und dem Neuritenwachstum. Wir zeigten durch Westernblotting mit einem Antikörper gegen eine konservierte Sequenz in der NOS, dass sich in den Entwicklungsstadien der Wanderungsbewegung im Darmgewebe eine höhere Konzentration des Enzyms als im zentralen Nervengewebe befindet (Knipp und Bicker 2009). Damit stellt der Darm eine potenzielle NO-Quelle dar, die das „Timing“ der Zellwanderung zur Innervierung seines Plexus über cGMP regulieren kann. Zur Untersuchung einer kausalen Beziehung zwischen dem NO/cGMP-System und der Zellmigration haben wir wieder in unserem Embryokultursystem die Signaltransduktion in Echtzeit chemisch manipuliert. Die Blockade der endogenen NO-Synthese verhindert die Migration der Mitteldarmneurone (Haase und Bicker 2003). Der sGC-Inhibitor ODQ blockiert ebenfalls dosisabhängig die Zellwanderung. Da ein spezifischer PKG-Inhibitor außerdem die Zellmigration hemmt, wirkt cGMP vermutlich über PKG als Effektorenzym. Die Verzögerung der Neuronenwanderung durch Hemmung der NO- oder cGMP-Synthese kann durch exogene Gabe von membranpermeablem cGMP oder der pharmakologischen Stimulation der sGC vollständig aufgehoben werden (Abbildung 5E-J). Die Rescue-Experimente zeigen, dass die NO/ cGMP-Signaltransduktion essenziell für die Regulation der Zellwanderung ist und in vivo ein gewisser cGMP-Spiegel für die Beweglichkeit der Mitteldarmneurone benötigt wird. Da die Inhibition der NOS oder sGC keine Fehler in der Navigation auf den Wanderungsstraßen hervorruft, gibt es bei dieser Form der Zellmigration keine Evidenz für eine richtungsweisende Funktion der NO-Freisetzung. NO wirkt also permissiv aber nicht instruktiv auf die gerichtete Zellwanderung.

Um eine transzelluläre Diffusion von NO aus den Darmzellen zu den wandernden Mitteldarmneuronen zu untersuchen, wurde Hämoglobin als NO-Scavenger (Abbildung 1) eingesetzt. Da exogen zugesetztes Hämoglobin durch seine Molekülgröße bedingt in der extrazellulären Gewebeflüssigkeit verbleibt, ist sein Hemmeffekt auf die Zellwanderung der Mitteldarmneuronen als Evidenz für eine interzelluläre NO/cGMP-Signalübermittlung zu werten (Knipp und Bicker 2009).

Es ist momentan noch nicht im Detail bekannt, wie die NO/cGMP/ PKG-Kaskade das Wanderverhalten der Mitteldarmneurone beeinflusst. Die Zellwanderung wird von einer Umorganisation des Zytoskeletts begleitet und hängt unter anderem von Kräften ab, die bei der Polymerisation von Aktin an den Wachstumskegeln ansetzen. Die Aktinfilamente werden an dem unterliegenden Substrat angeheftet und tragen so zur Translokation des Wachstumskegels bei. Wir haben filamentöses Aktin in den wandernden Mitteldarmneuronen mittels fluoreszenzgekoppeltem Phalloidin mikroskopisch visualisiert. In der Wanderphase sind Aktinfilamente hauptsächlich in den Zellfortsätzen und nicht im Zellkörper lokalisiert. Unter Bedingungen, in denen die Wanderung durch eine Blockade der NO/cGMP/PKG-Kaskade unterbunden wird, bildet sich ein dichtes Netzwerk von Aktinfilamenten im Zellkörper (Haase und Bicker 2003). Dieser Zustand kann auch durch eine Aktivierung der cAMP/Proteinkinase A-Signalkaskade erreicht werden. Die chemischen Manipulationen in Embryokultur zeigen, dass die NO/ cGMP-Signaltransduktion antagonistisch zur cAMP/PKA-Kaskade auf die Zellwanderung wirkt.

Neben den wandernden Neuronen wird der Mitteldarmplexus auch von zusätzlichen Neuriten innerviert, die am Vorderdarm positionierten kleinen Ganglienknoten des enterischen Nervensystems entspringen und größtenteils durch Serotonin-Immunzytochemie darstellbar sind (Stern et al. 2007). Das Neuritenwachtum dieser Neuronenpopulation folgt unmit-

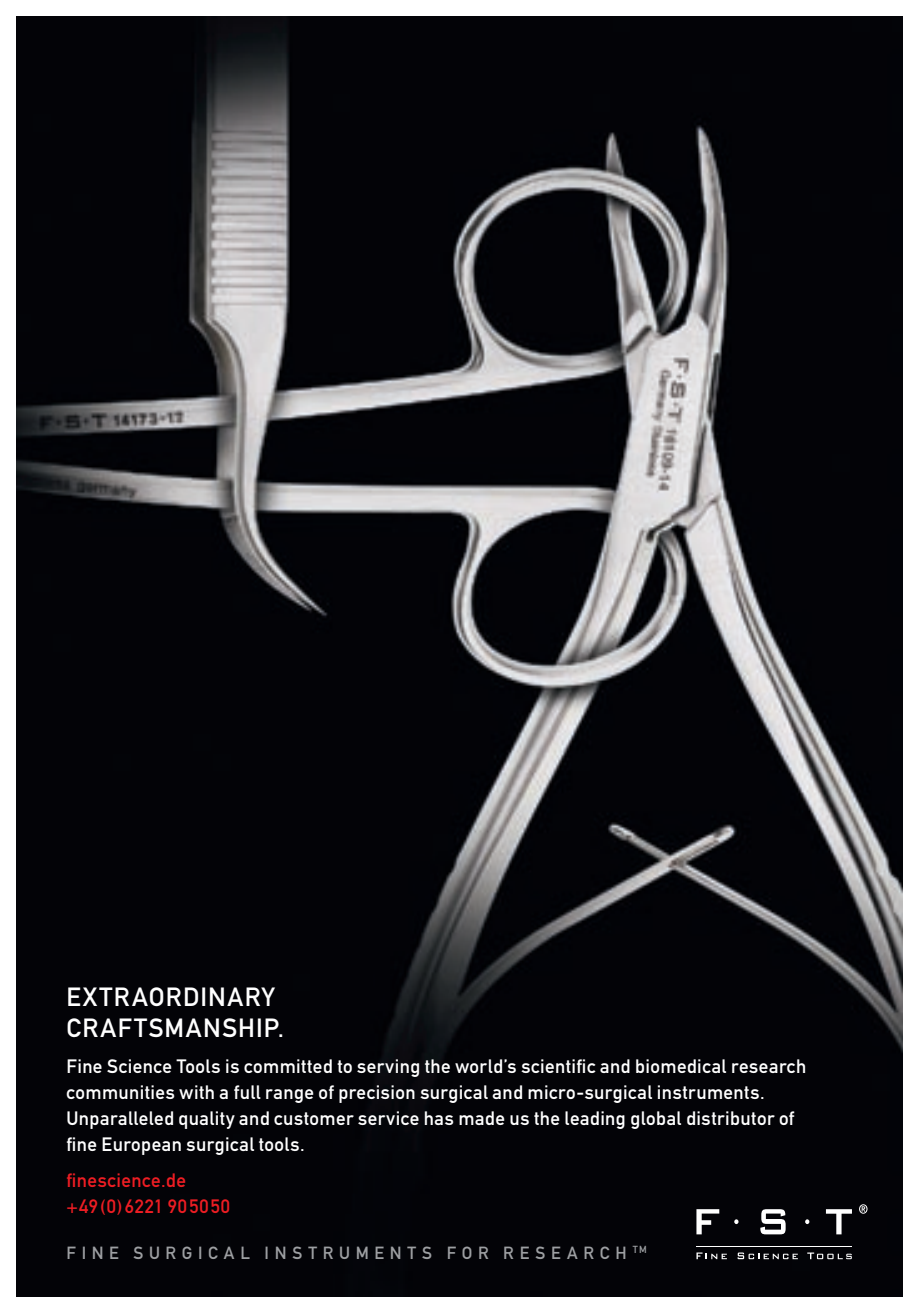




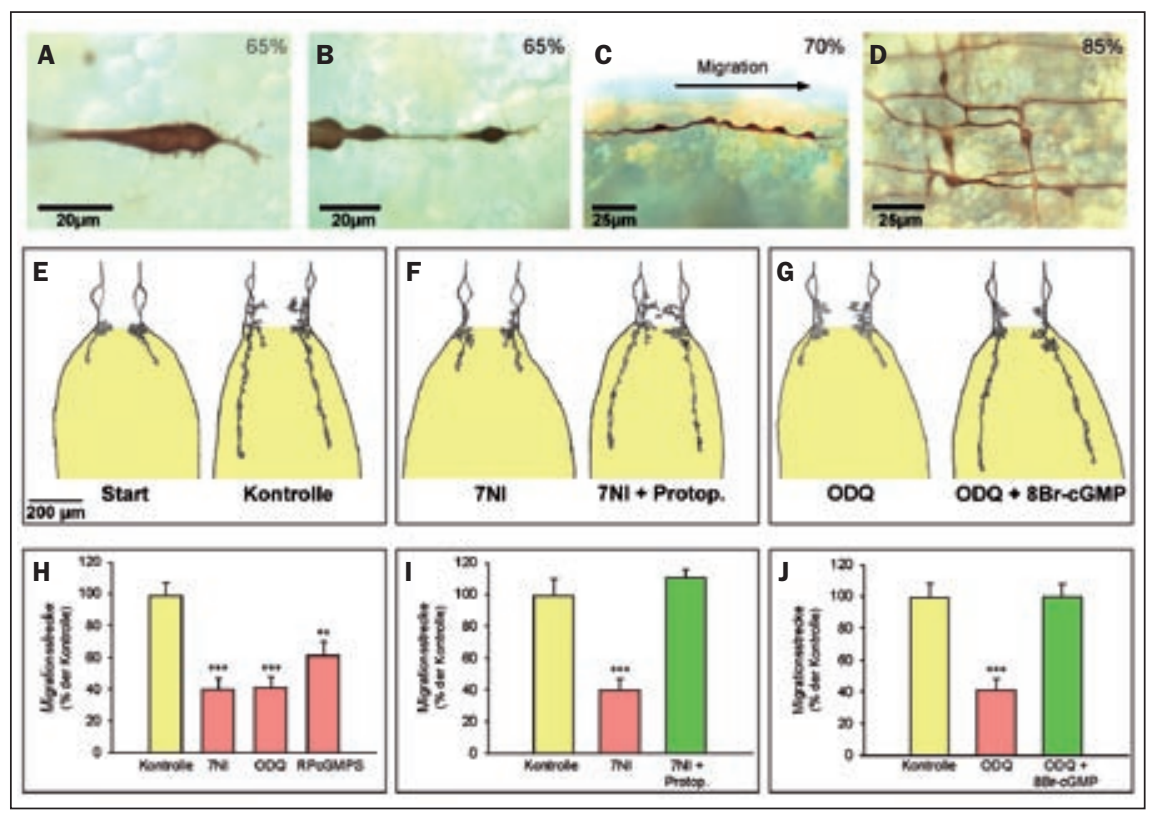

Abb. 5: Stickstoffmonoxid reguliert die Migration enterischer Neurone im Heuschreckenembryo. (A-D) NO-induzierte cGMP-Immunfärbung migrierender enterischer Neurone auf dem Mitteldarm in verschiedenen Entwicklungsstadien (angegeben in \%). Die führenden Neurone lassen immunreaktive Filopodien erkennen (A, B), werden von einer Kette von wandernden Neuronen gefolgt (C), die später die Zugstraßen verlassen und einen Plexus ausbilden (D). (E-H) Zeichnungen von cGMP-immunreaktiven enterischen Neuronen vor (Start) bzw. nach 24 Stunden in Kultur (alle anderen Zeichnungen). Es sind jeweils zwei von insgesamt vier Zugstraßen pro Darm gezeichnet. Zu Beginn des Experiments befinden sich die Neurone an der Grenze zwischen Vorderdarm und Mitteldarm (gelb) und wandern in $24 \mathrm{~h}$ ca. $300 \mu \mathrm{m}$ posterior (E). Inhibiton der NOS mit 7NI verhindert diese Migration, lässt sich aber durch direkte Stimulation der sGC mit Protoporphyrin IX wiederherstellen (F, I). Inhibition der SGC mit ODQ verhindert die Migration, lässt sich aber durch gleichzeitige Applikation des membranpermeablen cGMP-Analogons 8Br-cGMP wiederherstellen (G, J). Auch Inhibition der Proteinkinase G mit RPcGMPS hemmt die Migration (H). Die Daten in H-J sind Mittelwerte aus jeweils mindestens zehn Embryonen.

telbar der Kettenmigration der Mitteldarmneuronen auf den vier Wanderungsstraßen. Diese Neuriten zeigen keine NO-induzierte cGMP-Synthese. Dadurch konnten wir die Frage entscheiden, ob sich eine Blockade der NO-Synthese auch auf die Entwicklung von Neuronen auswirkt, die keine unmittelbaren Zielzellen der NO/cGMP-Kaskade sind. Wird die Wanderung der Mitteldarmzellen pharmakologisch mit 7NI gehemmt, so wird auch das Auswachsen der serotonergen Fasern verzögert (Stern et al. 2007). Damit ist gezeigt, dass Entwicklungsdefekte, die durch eine Störung der NO-Synthese bedingt sind, auch auf andere Zellen außerhalb der NO/ cGMP-Signalkaskade übersprechen.

\section{Verkehrsregulation der \\ Zellwanderung durch $\mathbf{C O}$}

Durch seinen einfachen Verlauf auf den geradlinigen Zugstraßen und die Mög- lichkeit, eine Vielzahl von Embryonen im gleichen Entwicklungsstadium in der Multiwellplatte mit chemischen Verbindungen zu inkubieren, bietet sich die Zellwanderung auf dem Mittelarmplexus als ein Screeningsystem für die expandierende neue Disziplin der ,chemischen Biologie“ an. Die bisher beschriebenen Versuche zeigten, dass wir den, ,loss of function“ Defekt in der Zellwanderung, durch Zugabe von Enzymaktivatoren stromabwärts des Blocks der NO-Signaltransduktionskette (Abbildung 1) kompensieren konnten. Wir haben inzwischen eine Reihe von Verbindungen auf ihre Wirksamkeit getestet, die Zellwanderung zu beschleunigen. Ein Überschuss des NOS-Substrats LArginin, verschiedene NO-Donoren und die Verstärkung der sGC-Enzymaktivität mit dem NO-unabhängigen Aktivator YC-1 führen jedoch zu keiner statistisch signifikanten Beschleunigung der Zellwan- derung. Überraschenderweise konnten wir jedoch mit niedrigen Konzentrationen von bestimmten Metalloporphyrinen, die als Hämoxigenase-Inhibitoren wirken, eine robuste Beschleunigung der Zellwanderung erzielen (Knipp und Bicker 2009). Hämoxigenase katalysiert den Abbau von Häm zu Biliverdin, einem Prozess, bei dem Kohlenstoffmonoxid (CO) als Beiprodukt frei wird (Boening und Snyder 2003). Umgekehrt kann man durch Applikation eines Hämoxigenase-Substrats oder von CO-Donoren auf den Insektenembryo die Zellwanderung wieder verzögern. Durch Arbeiten, die im Labor von Solomon Snyder ihren Ausgang nahmen, steht CO unter gut begründetem Verdacht, ein weiterer gasförmiger Botenstoff $\mathrm{zu}$ sein, der im Nervensystem aktivitätsabhängig gebildet wird. Wir konnten am Insektenembryo zeigen, dass die wandernden Mitteldarmneuronen eine transiente Immunreaktivität gegen die neuronale Isoform der Hämoxigenase zeigen (Knipp und Bicker 2009). Da die pharmakologische Hemmung der Hämoxigenase zu einem, gain of function“ in der Zellwanderung führt, postulieren wir, dass eine endogene CO-Produktion in den Mitteldarmneuronen die Zellwanderung verzögert. Sowohl NO als auch CO binden an $\mathrm{sGC}$, aber bei der Stimulation der enzymatischen cGMP-Bildung ist NO um Größenordnungen effizienter als CO. Daher könnte über einen Kompetitionsmechanismus $\mathrm{CO}$ als endogener Koregulator die NO-induzierte cGMP-Bildung verringern und als Bremspedal die Wanderung der Mitteldarmneuronen dem Verkehrsfluss anpassen.

\section{Regulation der Zellmotilität im Nervensystem von Vertebraten}

Da NO bereits als ein essenzieller Mediator der Zellwanderung in der glatten Muskulatur, in Epithelien und bei Makrophagen identifiziert wurde (Brown et al. 1999; Elferink und VanUffelen 1996), ist es im Rückblick eigentlich nicht verwunderlich, dass dieser gasförmige Botenstoff auch als Regulator der Zellmotilität bei der Entwicklung in Evertebratennervensystemen eingesetzt wird. Bei einem bestimmten NO synthetisierenden Neuron der Schnecke Helisoma orchestriert die NO/cGMPSignalbahn das Neuritenwachstum und eine Verlängerung der Filopodien auf dem Wachstumskegel (Trimm und Rehder 2004). Diese präzise Funktion steht vermutlich im Zusammenhang mit einer verbesserten Orientierung des Wachstumskegels an Entscheidungspunkten der 


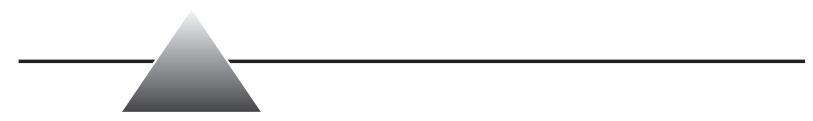

Wegfindung. Erstaunlicherweise reguliert NO nicht nur die Zellwanderung in tierischem Gewebe, sondern besitzt auch beim Spitzenwachstum von pflanzlichen Pollenschläuchen eine Leitfunktion (Prado et al. 2004). Da viele der Signalkaskaden der axonalen Navigation zwischen Evertebraten und Vertebraten in der Evolution konserviert sind, wollen wir uns der Frage zuwenden, ob NO auch bei den massiven Neuronenwanderungen bei der Entwicklung des Wirbeltiergehirns eine Rolle spielt.

Es gibt in der Tat eine Reihe von Hinweisen, die diese Vorstellung unterstützen. Neuroanatomische Studien mit Markern gegen NOS und sGC deuten darauf hin, dass die wandernden Neuroblasten des rostralen migratorischen Stroms potenzielle Zielzellen für NO Signale sind (Guiterrez-Mecinas et al. 2007). Bei der Entwicklung des menschlichen Rückenmarks exprimieren definierte Untergruppen von Interneuronen auf ihrer Wanderung zum Zielgebiet NOS (Foster und Phelps 2000). Für die transiente Expression von Enzymen der NO/ cGMP/PKG-Kaskade in kritischen Phasen der Hirnentwicklung lassen sich noch weitere Beispiele anführen.

Die Bildung des Zerebellums ist charakterisiert durch eine charakteristische Wanderung der Körnerzellen von der externen zu der internen Körnerzellschicht. Eine pharmakologische Blockade der NOS oder der Einsatz des NO-Scavengers Hämoglobin in einem Kultursystem für Zerebellumschnitte hemmen die Wanderung der Körnerzellen (Tanaka et al. 1994). Diese Experimente sind Evidenz für eine Rolle von endogener NO-Synthese bei Wanderungsprozessen der Körnerzellen. Knockout-Mäuse, die in einer Isoform der PKG defizient sind, zeigen Störungen in der Entwicklung des Neokortex, die sich auf abnormale neuronale Wanderung zurückführen lassen und damit die cGMP/PKG-Signalbahn implizieren (Demyaneko et al. 2005). Kürzlich wurde ein besonders spektakuläres Beispiel für strukturelle Plastizität im Nervensystem entdeckt, das auf einer gerichteten Orientierung von präsynaptischen Zellfortsätzen beruht. Die NO-Freisetzung aus hippocampalen Neuronen induziert retrograd eine multiaxonale Innervation der dendritischen Spines (Nikonenko et al. 2008).

Lassen sich nun die Ergebnisse vom Entwicklungsmodell des Insektenembryos auch auf die menschliche Gehirnentwicklung übertragen? Um diese Frage ansatzweise experimentell anzugehen, haben wir Neurosphärenkulturen einer humanen Teratokarzinomzelllinie (Ntera2) etabliert, die sich unter dem Einfluss von Retinsäure in postmitotische Neuronen differenzieren (Paquet-Durand und Bicker 2007). Werden die Neurosphären auf adhärentem Substrat plattiert, so migrieren die Vorläuferzellen aus der Peripherie aus und lassen sich videomikroskopisch bezüglich ihrer Wanderstrecke quantifizieren. Wir konnten durch Blockade wie durch Aktivierungsexperimente eine positive Wirkung von $\mathrm{NO} /$ cGMP/PKG-Signaltransduktion auf die Motilität neuronaler Vorläuferzellen in der Zellkultur nachweisen (Tegenge und Bicker 2008). Diese Ergebnisse deuten darauf hin, dass sowohl bei der Entwicklung des Grashüpfer- wie des menschlichen Nervensystems NO als Regulator für Mechanismen der Zellwanderung dient.

\section{Literatur}

Garthwaite, J. (2008): Concepts of neural nitric oxide-mediated transmission. Eur J Neurosci 27: 2783-2802.

Electronic Instruments

for the Life Sciences

\section{The "Swiss Army Knife" of Electrophysiology}

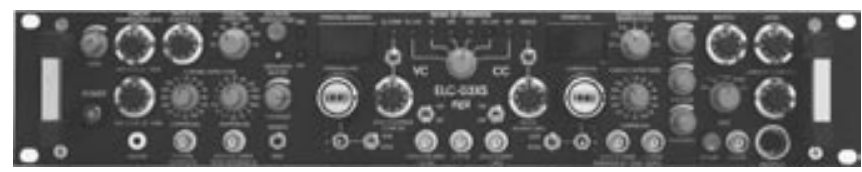

\section{ELC-03XS}

Suitable for extracellular recordings with high gain, juxtasomal filling of dyes or DNA, intracellular recordings, whole-cell patch clamp in true CC or VC mode, single cell stimulation and electroporation, amperometry and voltammetry, and iontophoresis

\section{Versatile Current Clamp Amplifier}

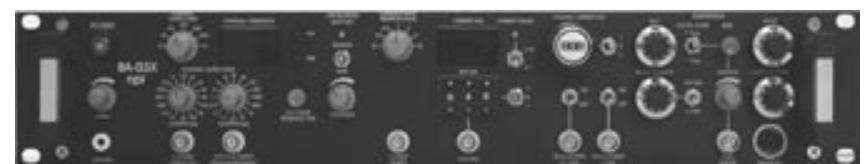

BA-03X

Suitable for intracellular recordings, extracellular recordings with high gain and electroporation

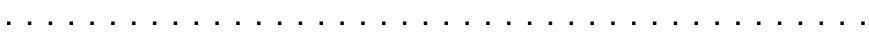

\section{Uscientifica}

PatchStar

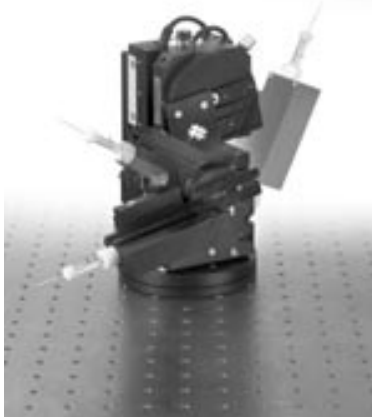

The Patchstar is a high precision, stable and motorized manipulator, with the ability to move in $X Y Z$ and a virtual approach axis. It offers a resolution of $20 \mathrm{~nm}, 4$ axes of motion (three real, one virtual) and is electrically silent.

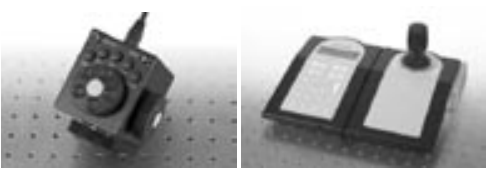

Other npi electronic instruments

Single Electrode voltage clamp amplifiers

Two Electrode voltage clamp amplifiers Temperature control systems Bridge-/Intracellular amplifiers Extracellular amplifiers EPMS modular system, Bessel filters Drug application systems

Data acquisition hard and software

Voltammetric / amperometric amplifiers ALA Scientific perfusion systems and accessories

EXFO Burleigh micropositioners and mounts Scientifica micropositioners and mounts

npi electronic $\mathrm{GmbH}$

Hauptstrasse 96, D-71732 Tamm, Germany Phone + 49 (0)7141-9730230; Fax: + 49 (0)7141-9730240 suppor@@npielectronic.com; http://www.npielectronic.com 
Haase, A. und Bicker, G. (2003): Nitric oxide and cyclic nucleotides are regulators of neuronal migration in an insect embryo. Development 130: 3977-3987.

Knipp, S. und Bicker, G. (2009): Regulation of enteric neuron migration by the gaseous messenger molecules CO and NO. Development 136: 85-93.

Seidel, C. und Bicker, G. (2000): Nitric oxide and cGMP influence axonogenesis of antennal pioneer neurons. Development 127: 4541-4549.

Stern, M., Knipp, S. und Bicker, G. (2007): Embryonic differentiation of serotonincontaining neurons in the enteric nervous system of the locust (Locusta migratoria). $J$ Comp Neurol 501: 38-51.

Stern, M. und Bicker, G. (2008): Nitric oxide regulates axonal regeneration in an insect embryonic CNS. Dev Neurobiol 68: 295-308.

Wolf, G. (1997): Gasproduktion im Nervensystem: Stickoxid. Neuroforum 3/97: 100-107.

Eine vollständige Literaturliste kann bei den Autoren angefordert werden.

\section{Danksagung}

Wir danken der Deutschen Forschungsgemeinschaft für die finanzielle Unterstützung und allen Mitgliedern unseres Labors für ihre Beiträge zur Forschung an der NO-Signaltransduktion.

\section{Kurzbiografien}

Gerd Bicker: 1970-1976 Studium der Biologie in Freiburg. Promotion an der Universität Freiburg von 1976-1979 mit Aufenthalt am Weizmann Institute of Science. Anschließend Postdoc an der University of California, Santa Cruz und der University of Alberta, Edmonton von 1979-1982. Wissenschaftlicher Mitarbeiter und Oberassistent an der FU Berlin von 1982-1992. Von 1992-1997 Heisenberg-Stipendiat der DFG mit mehreren Forschungsaufenthalten am Department of Molecular and Cell Biology, University of California, Berkeley. Seit 1997
Professor für Zellbiologie an der Tierärztlichen Hochschule Hannover.

Michael Stern: 1986-1992 Studium der Biologie in Hamburg, 1995 Promotion an der Universität Hamburg, 1995-1997 Postdoc am Sussex Centre for Neurosciences, UK. Seit 1998 wissenschaftlicher Mitarbeiter in der Arbeitsgruppe Bicker an der Tierärztlichen Hochschule Hannover.

\section{Korrespondenzadresse}

\section{Prof. Gerd Bicker}

Abteilung Zellbiologie,

Physiologisches Institut,

Stiftung Tierärztliche Hochschule

Hannover

Bischofsholer Damm 15/102

30173 Hannover

Tel: $\quad+49(0) 511856-7272$

Fax: $\quad+49$ (0)511856-7687

E-Mail: gerd.bicker@tiho-hannover.de

\section{STELLENMARKT}

\section{UNIVERSITAT LEIPZIG}

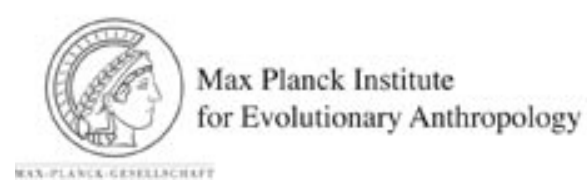

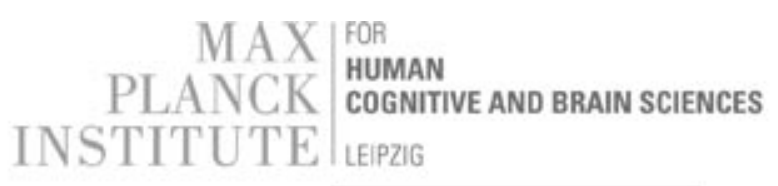

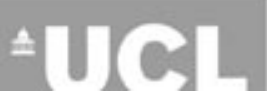

The International Max Planck Research School Neuroscience of Communication: Function, Structure, and Plasticity is based at the MPI for Human Cognitive and Brain Sciences, Leipzig (Germany) and the University of Leipzig, also involving the MPI for Evolutionary Anthropology, Leipzig and the Institute of Cognitive Neuroscience at UCL (UK).

The IMPRS offers a unique interdisciplinary graduate programme to study the functional, structural, and plastic bases of human communication. Besides behavioural work, the programme draws on elaborate modern imaging techniques including a 7-Tesla MRI scanner and a 306-channel MEG system.

The school invites applications for PhD scholarships.

Successful candidates will be accepted into one of the following four modules: (1) Verbal Communication (Language), (2) Non-verbal Communication (Action and Interaction), (3) Neuroscience, or (4) Methods. Requirement for successful candidates is a Master's (or qualified equivalent) degree in disciplines like computer science, linguistics, neurobiology, neurology, physics, psychiatry, psychology, or related fields.

Further requirements include outstanding academic performance; excellent oral and written English language skills; aptitude for original, independent, and creative work; performed research and published, or submitted for publication, results (desirable). Candidates near to completion may also submit applications, indicating the date of completion. Depending on qualification and background, we may consider applicants with a Bachelor's degree.

The application must be supported by a degree and school certificate, academic transcripts, a CV, three names and email addresses of academic referees, and a personal statement explaining the candidate's motivation and reasons for pursuing a PhD at the IMPRS. Applications should indicate the preferred module into which the candidate wishes to be accepted, and specify the preferred supervisor. Applications are to be submitted in electronic format only until 31 March 2009.

All admitted students receive a scholarship for the duration of 3 years. The graduate programme will start with the Summer Semester 2009 at the University of Leipzig (1 April 2009). The language of the IMPRS is English. Further information under: http://imprs-neurocom. mpg.de.

Contact: Dr Antje Holländer, IMPRS Co-ordinator, e-mail: imprs@cbs.mpg.de, phone: +49 (0)341 9940-2261 